\title{
OA-019 NEW POSSIBILITIES FOR THE DEVELOPMENT OF A COMBINED VACCINE AGAINST ETEC AND SHIGELLA
}

Richard Walker. PATH, United States of America

10.1136/bmjgh-2016-000260.26

Background Together, enterotoxigenic Escherichia coli (ETEC) and Shigella contribute greatly to the mortality and the morbidity due to diarrhoeal diseases, including a number of negative lifetime health impacts. Vaccines represent a reasonable option to reduce this burden.

Vaccine candidates PATH has reviewed the landscape of vaccine candidates for these diseases and identified two candidates to be moved towards licensure in the near-term as a combined ETEC and Shigella vaccine for use on an Expanded Programme on Immunisation (EPI) schedule.

ETVAX One candidate is a formalin-inactivated ETEC vaccine (ETVAX) consisting of four E. coli preparations, each engineered to hyper-produce the CFA/1, CS3, CS5, and CS6 antigens of ETEC. In addition, the vaccine contains a cholera $\mathrm{B}$ subunit modified to be more cross-reactive with the B subunit of ETEC. ETVAX is co-administered with a double-mutant of the ETEC heat-labile toxin (dmLT), which serves as a potent mucosal adjuvant.

TSWC The other candidate includes formalin-killed S. flexneri $2 \mathrm{a}$ and $3 \mathrm{a}$ and $S$. sonnei prepared as a trivalent vaccine, called TSWC. A prototype of TSWC, S. flexneri 2a, was administered to North American volunteers and found to be safe and immunogenic; it is now currently in a challenge trial.

Looking ahead Early 2017 in a phase I trial, we will test TSWC given alone and co-administered with ETVAX. ETVAX given alone exceeded expectations for immunogenicity in Swedish volunteers and is projected to be evaluated for safety and early efficacy in Finnish travellers to Benin in early 2017. ETVAX is also currently being tested in a descending-age trial in 


\section{Abstracts}

Bangladesh to determine the optimum safe dose of vaccine and adjuvant to be given to infants as young as 6 to 10 weeks of age. We also hope that the dmLT will have a dose-sparing effect on the vaccine given to this target population. 